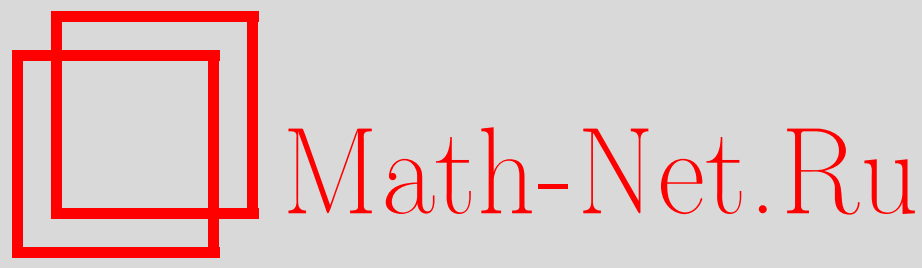

И. А. Баталин, П. М. Лавров, Конверсия связей второго рода и решение условий нулевой кривизны в теории геометрического квантования, ТМФ, 2016, том 187, номep 2, 200-212

DOI: https://doi.org/10.4213/tmf9007

Использование Общероссийского математического портала Math-Net.Ru подразумевает, что вы прочитали и согласны с пользовательским соглашением http: //www . mathnet.ru/rus/agreement

Параметры загрузки:

IP : 3.85 .7 .115

26 апреля 2023 г., 14:11:55

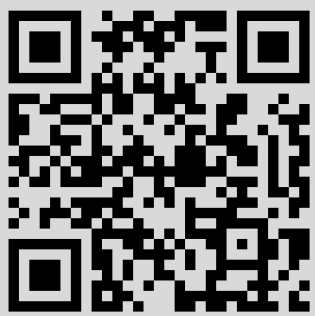




\section{КОНВЕРСИЯ СВЯЗЕЙ ВТОРОГО РОДА И РЕШЕНИЕ УСЛОВИЙ НУЛЕВОЙ КРИВИЗНЫ В ТЕОРИИ ГЕОМЕТРИЧЕСКОГО КВАНТОВАНИЯ}

В подходе к геометрическому квантованию, основанном на конверсии связей второго рода, решаются соответствующие нелинейные условия нулевой кривизны для расширенного симплектического потенциала. Из условий нулевой кривизны выведены новые линейные уравнения для расширенного симплектического потенциала. Показано, что решения новых линейных уравнений также удовлетворяют условию нулевой кривизны. Предложено функциональное решение этих новых линейных уравнений и получено соответствующее представление в форме функционального интеграла по путям. Исследование проведено для общего случая фазового суперпространства, когда бозонные и фермионные координаты присутствуют на равном основании.

Ключевые слова: симплектический потенциал, связи второго рода, метод конверсии.

DOI: $10.4213 / \operatorname{tmf} 9007$

\section{1. ВВЕДЕНИЕ}

Мы счастливы возможности представить статью в честь 75-летия нашего друга и коллеги - профессора Игоря Викторовича Тютина. Мы работали вместе с Игорем Тютиным много лет, и мы оценили в полной мере его экстраординарный научный потенциал, а также его блестящие личные качества. Мы сердечно желаем Игорю Тютину много новых научных достижений вместе с дальнейшими всевозможными успехами в личной жизни.

Фундаментальная концепция квантования, предложенная Березиным [1], имеет нетривиальную проекцию на геометрическое квантование [2]-[5], формализм Баталина-Фрадкина-Вилковыского (БФВ) [6], [7] и деформационное квантование [8]-[10].

Работа И.А. Баталина частично поддержана РФФИ (гранты №14-01-00489, 14-02-01171). Работа П. М. Лаврова поддержана Министерством образования и науки Российской Федерации (грант № 3.867.2014/K).

*Физический институт им. П. Н. Лебедева РАН, Москва, Россия. E-mail: batalin@lpi.ru

${ }^{\dagger}$ Томский государственный педагогический университет, Томск, Россия.

E-mail: lavrov@tspu.edu.ru 
Хорошо известно, что конверсия связей второго рода служит одним из наиболее мощных современных подходов к геометрическому квантованию. Стандартный сценарий метода конверсии [11]-[16] (см. также обзор [17] и дальнейшее развитие в [18]-[20]) формулируется следующим образом. Исходным является некоторое фазовое многообразие со сложной нелинейной скобкой Пуассона. Затем вводятся новые импульсы как канонически сопряженные исходным фазовым переменным, которые рассматриваются теперь как коммутирующие между собой. В то же самое время вводятся связи второго рода, приравнивающие новые импульсы к компонентам симплектического потенциала. Затем вводятся дополнительные степени свободы (переменные конверсии) [14], [21] для преобразования связей второго рода в связи первого рода. Скобки Пуассона для переменных конверсии объявляются постоянными. Получающиеся после конверсии связи первого рода приравнивают новые импульсы к компонентам расширенного симплектического потенциала, зависящим теперь также от переменных конверсии. Обычно предполагается, что эти связи первого рода коммутируют между собой (абелева конверсия). Тем самым их коммутационные соотношения имеют форму условия нулевой кривизны. Эти условия нулевой кривизны являются фактически нелинейными уравнениями, которые трудно технически разрешить для расширенного симплектического потенциала.

В настоящей статье мы выводим новые, линейные, уравнения для расширенного симплектического потенциала, умножая условия нулевой кривизны на исходные фазовые переменные. Затем мы показываем, что решения этих линейных уравнений удовлетворяют также нелинейным условиям нулевой кривизны. Ситуация здесь очень похожа на случай уравнения Маурера-Картана в стандартной теории групп. В заключение мы приводим функциональное решение для новых линейных уравнений и затем выводим соответствующее представление в форме функционального интеграла по путям.

\section{2. КЛАССИЧЕСКАЯ МЕХАНИКА В ОБЩЕМ ПОЛОЖЕНИИ}

Пусть $Z^{A}, \varepsilon\left(Z^{A}\right)=\varepsilon_{A}$, - координаты исходного фазового пространства в гамильтоновом формализме, $V_{A}(Z), \varepsilon\left(V_{A}\right)=\varepsilon_{A}$, - симплектический потенциал, а $S$ действие с исходным гамильтонианом $H=H(Z)$,

$$
S=\int d t \mathcal{L}, \quad \mathcal{L}=V_{A} \partial_{t} Z^{A}-H .
$$

Произвольная вариация $\delta Z^{A}$ дает уравнения движения

$$
\omega_{A B} \partial_{t} Z^{B}-\partial_{A} H=0,
$$

где $\omega_{A B}-$ симплектическая метрика,

$$
\omega_{A B}=\partial_{A} V_{B}+\partial_{B} V_{A}(-1)^{\left(\varepsilon_{A}+1\right)\left(\varepsilon_{B}+1\right)} .
$$

Из (2.3) следует, что

$$
\partial_{C} \omega_{A B}(-1)^{\left(\varepsilon_{C}+1\right) \varepsilon_{B}}+\text { cyclic perm. }(A, B, C)=0 .
$$

В дальнейшем мы предполагаем, что метрика (2.3) является обратимой, и обозначаем обратную к ней через

$$
\omega^{A B}=-\omega^{B A}(-1)^{\varepsilon_{A} \varepsilon_{B}},
$$


так что из (2.4) мы имеем

$$
\omega^{A D} \partial_{D} \omega^{B C}(-1)^{\varepsilon_{A} \varepsilon_{C}}+\text { cyclic perm. }(A, B, C)=0 .
$$

Умножая (2.3) слева на $Z^{A}$, мы получаем для $V_{B}$ уравнение

$$
\left(Z^{A} \partial_{A}+1\right) V_{B} \mathcal{F}_{0} \overleftarrow{\partial}_{B}=Z^{A} \omega_{A B}
$$

с произвольной функцией $\mathcal{F}_{0}=Z^{A} V_{A}(-1)^{\varepsilon_{A}}$. В свою очередь, из $(2.7)$ следует, что лагранжиан в (2.1) можно переписать в виде

$$
\mathcal{L}=Z^{A} \bar{\omega}_{A B} \partial_{t} Z^{B}-H+\partial_{t} \chi
$$

где $\chi$ - произвольная функция, а метрика $\bar{\omega}$ определяется уравнением

$$
\left(Z^{C} \partial_{C}+2\right) \bar{\omega}_{A B}=\omega_{A B}
$$

Из (2.2) затем следует, что

$$
\partial_{t} Z^{A}-\omega^{A B} \partial_{B} H=0
$$

Теперь, пусть $P_{A}, \varepsilon\left(P_{A}\right)=\varepsilon_{A}$, - импульсы, канонически сопряженные $Z^{A}$. Тогда действие (2.1) переписывается в виде

$$
\mathcal{L}=P_{A} \partial_{t} Z^{A}-H-\Theta_{A} \lambda^{A}
$$

где $\lambda^{A}, \varepsilon\left(\lambda^{A}\right)=\varepsilon_{A}$, являются множителями Лагранжа,

$$
\Theta_{A}=P_{A}-V_{A}
$$

представляют собой связи второго рода,

$$
\left\{\Theta_{A}, \Theta_{B}\right\}=\omega_{A B}(-1)^{\varepsilon_{A}}, \quad\left\{Z^{A}, P_{B}\right\}=\delta_{B}^{A} .
$$

Определим для любых функций $F(Z), G(Z)$ скобку Дирака, соответствующую связям второго рода (2.12):

$$
\{F, G\}_{\mathrm{D}}=\{F, G\}-\left\{F, \Theta_{A}\right\} \omega^{A B}(-1)^{\varepsilon_{B}}\left\{\Theta_{B}, G\right\},
$$

где мы использовали (2.13). Так как $\{F(Z), G(Z)\}=0$, то из (2.14) немедленно следует, что

$$
\{F, G\}_{\mathrm{D}}=F \overleftarrow{\partial}_{A} \omega^{A B} \vec{\partial}_{B} G
$$

В силу (2.6) мы имеем

$$
\left\{F,\{G, H\}_{\mathrm{D}}\right\}_{\mathrm{D}}(-1)^{\varepsilon_{F} \varepsilon_{H}}+\text { cyclic perm. }(F, G, H)=0 .
$$

Таким образом, скобка Дирака (2.15) воспроизводит “криволинейную" скобку Пуассона, отвечающую метрике (2.5). В терминах скобки Дирака (2.15) уравнения движения (2.10) переписываются как

$$
\partial_{t} Z^{A}=\left\{Z^{A}, H\right\}_{\mathrm{D}} .
$$


Ротор в правой части (2.3) является пока абелевым. Пусть теперь $\Phi^{A}, \varepsilon\left(\Phi^{A}\right)=\varepsilon_{A}$, суть переменные конверсии, чьи отличные от нуля скобки Пуассона определяются соотношениями

$$
\left\{\Phi^{A}, \Phi^{B}\right\}=\eta^{A B}=\text { const. }
$$

Метрика (2.18) предполагается обратимой, и ее обращение обозначается как $\eta_{A B}$. В духе общей идеологии метода конверсии определим новые связи

$$
T_{A}=P_{A}-\mathcal{V}_{A}(Z, \Phi)
$$

и потребуем, чтобы они были абелевыми связями первого рода,

$$
\left\{T_{A}, T_{B}\right\}=\left(\partial_{A} \mathcal{V}_{B}+\partial_{B} \mathcal{V}_{A}(-1)^{\left(\varepsilon_{A}+1\right)\left(\varepsilon_{B}+1\right)}\right)(-1)^{\varepsilon_{A}}+\left\{\mathcal{V}_{A}, \mathcal{V}_{B}\right\}=0
$$

Эти уравнения следует решать относительно $\mathcal{V}_{A}(Z, \Phi)$ при граничном условии

$$
\left.\mathcal{V}_{A}(Z, \Phi)\right|_{\Phi=0}=V_{A}(Z)
$$

В линейном порядке по $\Phi^{A}$,

$$
\mathcal{V}_{A}=V_{A}-V_{A B} \Phi^{B}+\mathcal{O}\left((\Phi)^{2}\right)
$$

соотношения инволюции (2.20) дают

$$
V_{A C} \eta^{C D} V_{B D}(-1)^{\left(\varepsilon_{B}+1\right) \varepsilon_{D}}=-\omega_{A B}(-1)^{\varepsilon_{A}} .
$$

Таким образом, $V_{A B}$ служит в качестве "матричнозначного квадратного корня" из метрики (2.13). Коэффициенты высших порядков разложения по Ф расширенного симплектического потенциала $\mathcal{V}_{A}$ являются высшими структурными функциями расслоения, генерируемого $V_{A B}$. Уравнение “квадратного корня" (2.23) естественным образом определяет свойство ковариантного постоянства метрики (2.3),

$$
\nabla_{C}(\Delta) \omega_{A B}=0=\partial_{C} \omega_{A B}-\Delta_{C A}^{D} \omega_{D B}(-1)^{\varepsilon_{A}+\varepsilon_{D}}-\Delta_{C B}^{D} \omega_{A D}(-1)^{\varepsilon_{A}\left(\varepsilon_{B}+\varepsilon_{D}\right)}
$$

где связность далекого параллелизма в терминах $V_{A B}$ и обратной к ней $V^{A B}$ определяется как

$$
\Delta_{C A}^{D}=\left(\partial_{C} V_{A B}\right) V^{B D}
$$

Соответствующая кривизна равна нулю,

$$
\begin{aligned}
& \partial_{E} \Delta_{C A}^{D}-\partial_{C} \Delta_{E A}^{D}(-1)^{\varepsilon_{E} \varepsilon_{C}}+ \\
& \quad+\Delta_{C A}^{F} \Delta_{E F}^{D}(-1)^{\varepsilon_{E}\left(\varepsilon_{C}+\varepsilon_{A}+\varepsilon_{F}\right)}-\Delta_{E A}^{F} \Delta_{C F}^{D}(-1)^{\varepsilon_{C}\left(\varepsilon_{A}+\varepsilon_{F}\right)}=0
\end{aligned}
$$

так что связность (2.25) является интегрируемой.

Будем искать решение уравнения (2.20) в виде разложения по $\Phi$ :

$$
\begin{gathered}
T_{A}=\sum_{n=0}^{\infty} T_{A}^{(n)}, \quad T_{A}^{(n)} \sim \Phi^{n}, \\
T_{A}^{(0)}=P_{A}-V_{A}, \quad T_{A}^{(n)}=-\mathcal{V}_{A}^{(n)}, \quad n \geqslant 1 .
\end{gathered}
$$


Рекуррентная формула для такого решения следует из теоремы в работе [22],

$$
T_{A}^{(n+1)}=-(n+2)^{-1} \Phi^{D} \eta_{D C} V^{C B} W_{B A}^{(n)}+\left\{T_{A}^{(1)}, \mathcal{K}^{(n+2)}\right\}_{(\Phi)}, \quad n \geqslant 1,
$$

где введены обозначения

$$
\begin{gathered}
\mathcal{K}^{(n+2)} \sim \Phi^{(n+2)}, \\
W_{A B}^{(1)}=\left\{T_{A}^{(0)}, T_{B}^{(1)}\right\}_{(P, Z)}-\left\{T_{B}^{(0)}, T_{A}^{(1)}\right\}_{(P, Z)}(-1)^{\varepsilon_{A} \varepsilon_{B}}, \\
W_{A B}^{(n)}=\sum_{m=0}^{n}\left\{T_{A}^{(n-m)}, T_{B}^{(m)}\right\}_{(P, Z)}+\sum_{m=0}^{n-2}\left\{T_{A}^{(n-m)}, T_{B}^{(m+2)}\right\}_{(\Phi)}, \quad n \geqslant 2 .
\end{gathered}
$$

$\eta_{A B}$ и $V^{A B}$ являются обратными к $\eta^{A B}$ и $V_{A B}$ соответственно. Кроме того, мы обозначили через $\{\cdot, \cdot\}_{(P, Z)}$ и $\{\cdot, \cdot\}_{(\Phi)}$ скобки Пуассона в секторах $P, Z$ и $\Phi$ соответственно. Частный случай рекуррентной формулы (2.29) при $n=1$ имеет вид

$$
T_{A}^{(2)}=-\frac{1}{3} \Phi^{D} \eta_{D C} V^{C B}\left(\left\{P_{B}, V_{A E}\right\}-\left\{P_{A}, V_{B E}\right\}(-1)^{\varepsilon_{A} \varepsilon_{B}}\right) \Phi^{E}-\left\{\mathcal{V}_{A}^{(1)}, \mathcal{K}^{(3)}\right\}_{(\Phi)}
$$

Ротор в правой части (2.20) определенно имеет структуру, характерную для теории Янга-Миллса, так что уравнение для тензора кривизны (2.20) является типичным условием нулевой кривизны. Тем самым решение для $\mathcal{V}_{A}$ имеет обычную структуру формы Картана, которая, в свою очередь, генерирует неабелевы упорядоченные экспоненты.

Теперь рассмотрим расширенный гамильтониан $\mathcal{H}$, определенный с помощью соотношений

$$
\left\{\mathcal{H}, T_{A}\right\}=0,\left.\quad \mathcal{H}\right|_{\Phi=0}=H,
$$

где $T_{A}$ - абелевы связи (2.19) после конверсии. Так же как и в (2.27), будем искать решение (2.32) в форме разложения по $\Phi$,

$$
\mathcal{H}=\sum_{n=0}^{\infty} \mathcal{H}^{(n)}, \quad \mathcal{H}^{(n)} \sim \Phi^{n}, \quad \mathcal{H}^{(0)}=H
$$

Рекуррентная формула для такого решения следует также из теоремы в [22]:

$$
\mathcal{H}^{(n+1)}=-(n+1)^{-1} \Phi^{A} \eta_{A B} V^{B C} W_{C}^{(n)}, \quad n \geqslant 0,
$$

где введены обозначения

$$
\begin{aligned}
W_{A}^{(0)}= & \left\{T_{A}^{(0)}, \mathcal{H}^{(0)}\right\} \\
W_{A}^{(1)}= & \left\{T_{A}^{(1)}, \mathcal{H}^{(0)}\right\}+\left\{T_{A}^{(0)}, \mathcal{H}^{(1)}\right\}+\left\{T_{A}^{(2)}, \mathcal{H}^{(1)}\right\}_{(\Phi)}, \\
W_{A}^{(n)}= & \sum_{m=0}^{n}\left\{T_{A}^{(n-m)}, \mathcal{H}^{(m)}\right\}_{(P, Z)}+\sum_{m=0}^{n-2}\left\{T_{A}^{(n-m)}, \mathcal{H}^{(m+2)}\right\}_{(\Phi)}+ \\
& +\left\{T_{A}^{(n+1)}, \mathcal{H}^{(1)}\right\}_{(\Phi)}, \quad n \geqslant 2 .
\end{aligned}
$$


Таким образом, в частности, мы получаем при $n=0,1$

$$
\begin{aligned}
\mathcal{H}^{(1)}= & -\Phi^{A} \eta_{A B} V^{B C}\left\{P_{C}, H\right\}, \\
\mathcal{H}^{(2)}= & -\frac{1}{2} \Phi^{A} \eta_{A B} V^{B C}\left[-\left\{P_{C}, \Phi^{D} \eta_{D E} V^{E F}\left\{P_{F}, H\right\}\right\}+\right. \\
& +\frac{1}{3}\left(\Phi^{D} \eta_{D E} V^{E F}\left(\left\{P_{F}, V_{C G}\right\}-\left\{P_{C}, V_{F G}\right\}(-1)^{\varepsilon_{C} \varepsilon_{F}}\right)-\right. \\
& -(-1)^{\varepsilon_{C} \varepsilon_{E}} V^{E F}\left(\left\{P_{F}, V_{C D}\right\}-\right. \\
& \left.\left.\left.-\left\{P_{C}, V_{F D}\right\}(-1)^{\varepsilon_{C} \varepsilon_{F}}\right) \Phi^{D} \eta_{E G}\right) V^{G M}\left\{P_{M}, H\right\}\right] .
\end{aligned}
$$

Заметим, что расширение исходного фазового пространства, $(Z) \rightarrow(Z, P) \rightarrow$ $(Z, P, \Phi)$, можно сформулировать непосредственно на уровне действия $(2.1)$ :

$$
\begin{aligned}
S & \rightarrow \int d t\left[P_{A} \partial_{t} Z^{A}-H(Z)-\Theta_{A}(Z, P) \lambda^{A}\right] \rightarrow \\
& \rightarrow \int d t\left[P_{A} \partial_{t} Z^{A}+\frac{1}{2} \Phi^{A} \eta_{A B} \partial_{t} \Phi^{B}-\mathcal{H}(Z, \Phi)-T_{A}(Z, P, \Phi) \lambda^{A}\right] \rightarrow \\
& \rightarrow \mathcal{S}=\int d t\left[\mathcal{V}_{A}(Z, \Phi) \partial_{t} Z^{A}+\frac{1}{2} \Phi^{A} \eta_{A B} \partial_{t} \Phi^{B}-\mathcal{H}(Z, \Phi)\right],
\end{aligned}
$$

где $\lambda^{A}$ - множители Лагранжа к соответствующим связям. Абелевы связи первого рода $T_{A}(2.19)$ генерируют следующие калибровочные преобразования, оставляющие инвариантным действие $\mathcal{S}$ :

$$
\delta Z^{A}=\Xi^{A}(t), \quad \delta \Phi^{A}=-\eta^{A B}\left(\partial_{B} \mathcal{V}_{C}\right) \Xi^{C}(t), \quad \partial_{B}=\frac{\partial}{\partial \Phi^{B}}
$$

\section{3. КВАНТОВОЕ ОПИСАНИЕ}

Наше рассмотрение распространяется на случай квантовой механики простой заменой скобок Пуассона на (супер)коммутаторы. Последнее правило применяется непосредственно к (2.13), (2.18), (2.20) и т.д. как

$$
\{\cdot, \cdot\} \rightarrow(i \hbar)^{-1}[\cdot, \cdot] .
$$

Итак, операторно-значный аналог (2.20) представляется в виде следующих соотношений:

$$
\begin{gathered}
(i \hbar)^{-1}\left[T_{A}, T_{B}\right]=\left(\partial_{A} \mathcal{V}_{B}+\partial_{B} \mathcal{V}_{B}(-1)^{\left(\varepsilon_{A}+1\right)\left(\varepsilon_{B}+1\right)}\right)(-1)^{\varepsilon_{A}}+(i \hbar)^{-1}\left[\mathcal{V}_{A}, \mathcal{V}_{B}\right]=0 \\
\left.\mathcal{V}_{A}\right|_{\Phi=0}=V_{A}
\end{gathered}
$$

Выведем линейное дифференциальное уравнение, которое описывает структуру решения для $\mathcal{V}_{A}$. Умножая уравнение (3.2) на $Z^{A}(-1)^{\varepsilon_{A}}$ слева, мы получим (ср. с (2.7))

$$
\left(Z^{A} \partial_{A}+1\right) \mathcal{V}_{B}-\partial_{B} \mathcal{F}(-1)^{\varepsilon_{B}}+(i \hbar)^{-1}\left[\mathcal{F}, \mathcal{V}_{B}\right]=0
$$

где введено обозначение $\mathcal{F}=Z^{A} \mathcal{V}_{A}(-1)^{\varepsilon_{A}}$. Производя рескейлинг переменных $Z^{A}$ с бозонным параметром $t$,

$$
Z^{A} \rightarrow t Z^{A}
$$


мы переписываем (3.4) в виде

$$
\begin{gathered}
\partial_{t}\left(t \mathcal{V}_{B}\right)-\partial_{B} F(-1)^{\varepsilon_{B}}+(i \hbar)^{-1}\left[F, t \mathcal{V}_{B}\right]=0 \\
F=F(t, Z, \Phi)=Z^{A} \mathcal{V}_{A}(t Z, \Phi)(-1)^{\varepsilon_{A}}
\end{gathered}
$$

В свою очередь, из последнего уравнения следует, что

$$
t \mathcal{V}_{B}=\int_{0}^{t} d t^{\prime} T \exp \left\{\frac{i}{\hbar} \int_{t^{\prime}}^{t} F d t^{\prime \prime}\right\}\left(F \overleftarrow{\partial}_{B}\right) T^{*} \exp \left\{-\frac{i}{\hbar} \int_{t^{\prime}}^{t} F d t^{\prime \prime}\right\}
$$

где $T$ и $T^{*}$ - хронологическое и антихронологическое упорядочивание соответственно. Вводя внешние источники $J_{A}(t)$ к упорядоченным по Вейлю операторам $\Phi^{A}$ (см. (3.14)), мы представим функциональное решение для левой хронологически упорядоченной экспоненты в (3.7) в виде

$$
\begin{aligned}
T \exp & \left\{\frac{i}{\hbar} \int_{t^{\prime}}^{t} F\left(t^{\prime \prime}, Z, \Phi\left(t^{\prime \prime}\right)\right) d t^{\prime \prime}\right\}=\left[\exp \left\{\frac{i}{\hbar} \int_{t^{\prime}}^{t} F\left(t^{\prime \prime}, Z, \frac{\hbar}{i} \frac{\delta}{\delta J\left(t^{\prime \prime}\right)}\right) d t^{\prime \prime}\right\} \times\right. \\
& \times \exp \left\{-\frac{i}{4 \hbar} \int_{t^{\prime}}^{t} d t^{\prime \prime} \int_{t^{\prime}}^{t} d t^{\prime \prime \prime} J_{A}\left(t^{\prime \prime}\right) \eta^{A B} \operatorname{sgn}\left(t^{\prime \prime}-t^{\prime \prime \prime}\right) J_{B}\left(t^{\prime \prime \prime}\right)(-1)^{\varepsilon_{B}}\right\} \times \\
& \left.\times \exp \left\{\frac{i}{\hbar} \int_{t^{\prime}}^{t} J_{A}\left(t^{\prime \prime}\right) \Phi^{A} d t^{\prime \prime}\right\}\right]\left.\right|_{J=0} .
\end{aligned}
$$

Производя функциональное Фурье-преобразование, из (3.8) можно вывести представление для левой упорядоченной экспоненты в (3.7) в виде функционального интеграла. Действуя таким образом, мы получаем следующее представление для решения (3.8):

$$
\int[D \Gamma] \exp \left\{\frac{i}{\hbar} \int_{t^{\prime}}^{t} d t^{\prime \prime}\left[\frac{1}{2} \Gamma^{A} \eta_{A B} \partial_{t^{\prime \prime}} \Gamma^{B}+F\left(t^{\prime \prime}, Z, \Gamma\left(t^{\prime \prime}\right)\right)\right]+\frac{i}{4 \hbar} \Gamma^{A}(t) \eta_{A B} \Gamma^{B}\left(t^{\prime}\right)\right\},
$$

где интегрирование ведется по виртуальным траекториям, удовлетворяющим граничному условию

$$
\Gamma^{A}(t)+\Gamma^{A}\left(t^{\prime}\right)=2 \Phi^{A} .
$$

Выражение (3.9) следует рассматривать как упорядоченную по Вейлю функцию операторов $\Phi^{A}$. Чтобы получить соответствующее функциональное решение для правой упорядоченной экспоненты в (3.7), следует изменить знак аргумента в первой и второй экспонентах в правой части в (3.8).

Мы утверждаем, что всякое решение уравнения (3.6) с произвольным $F$ удовлетворяет также уравнению (3.2). Легко вывести из (3.6) следующую задачу Коши:

$$
\partial_{t} X_{A B}=-(i \hbar)^{-1}\left[F, X_{A B}\right],\left.\quad X_{A B}\right|_{t=0}=0,
$$

где $X_{A B}$ определяется как

$$
X_{A B}=\left(\partial_{A} t \mathcal{V}_{B}+\partial_{B} t \mathcal{V}_{A}(-1)^{\left(\varepsilon_{A}+1\right)\left(\varepsilon_{B}+1\right)}\right)(-1)^{\varepsilon_{A}}+(i \hbar)^{-1}\left[t \mathcal{V}_{A}, t \mathcal{V}_{B}\right]
$$

с потенциалом $\mathcal{V}_{A}$, подвергнутым рескейлингу (3.5). В силу обычных аргументов из (3.11) следует, что

$$
X_{A B}=0 \quad \text { для любого } t,
$$


так что при $t=1$ мы приходим к уравнению (3.2). Оператор $F$ описывает произвол в решении.

Здесь и ниже мы полагаем, что выбран определенный тип нормального упорядочивания для всех операторнозначных функций. Для определенности мы выбираем упорядочивание по Вейлю. Тогда соответствующее звездочное умножение символов Вейля дается как

$$
\star=\exp \left\{\frac{i \hbar}{2} \overleftarrow{\partial}_{A} \eta^{A B} \vec{\partial}_{B}\right\}, \quad \partial_{A}=\frac{\partial}{\partial \Phi^{A}},
$$

$($ Operator $F)($ Operator $G) \rightarrow(\operatorname{Symbol} F) \star(\operatorname{Symbol} G)$,

$($ Operator $F)=\left.\exp \left\{\left(\right.\right.$ Operator $\left.\left.\Phi^{A}\right) \partial_{A}\right\}($ Symbol $F)\right|_{\Phi=0}$.

В квантово-механической ситуации полный унитаризующий гамильтониан после конверсии определяется как

$$
H_{\Psi}=\mathcal{H}+(i \hbar)^{-1}[\Psi, \Omega]
$$

где $\mathcal{H}$ есть решение следующей задачи Коши:

$$
\begin{aligned}
(i \hbar)^{-1}\left[\mathcal{H}, T_{A}\right] & =\mathcal{H} \overleftarrow{\partial}_{A}-(i \hbar)^{-1}\left[\mathcal{H}, \mathcal{V}_{A}\right]=0 \\
\left.\mathcal{H}\right|_{\Phi=0} & =H
\end{aligned}
$$

Калибровочный фермион имеет стандартный вид:

$$
\Psi=\bar{C}_{A} \chi^{A}+\overline{\mathcal{P}}_{A} \lambda^{A},
$$

генератор БРСТ-БФВ является типичным для случая абелевых связей:

$$
\Omega=C^{A}\left(P_{A}-\mathcal{V}_{A}\right)+\pi_{A} \mathcal{P}^{A}, \quad[\Omega, \Omega]=0,
$$

при стандартных коммутационных соотношениях релятивистского фазового пространства:

$$
\begin{gathered}
{\left[\lambda^{A}, \pi_{B}\right]=\left[C^{A}, \overline{\mathcal{P}}_{B}\right]=\left[\mathcal{P}^{A}, \bar{C}_{B}\right]=i \hbar \delta_{B}^{A},} \\
\varepsilon\left(\lambda^{A}\right)=\varepsilon\left(\pi_{A}\right)=\varepsilon_{A}, \quad \varepsilon\left(C^{A}\right)=\varepsilon\left(\overline{\mathcal{P}}_{A}\right)=\varepsilon\left(\mathcal{P}^{A}\right)=\varepsilon\left(\bar{C}_{A}\right)=\varepsilon_{A}+1 .
\end{gathered}
$$

Далее рассмотрим уравнение инволюции (3.18), аналогично тому, как мы делали раньше по отношению к уравнению (3.2). Умножая уравнение (3.18) на $Z^{A}$ справа и затем производя рескейлинг (3.5), мы выводим

$$
\partial_{t} \mathcal{H}-(i \hbar)^{-1}[\mathcal{H}, F]=0,
$$

где $F$ определено в (3.6). Мы утверждаем, что соотношения (3.6), (3.23) приводят к такому $\mathcal{H}$, которое удовлетворяет уравнению (3.18) с произвольным $F$. Легко вывести из уравнений (3.6), (3.23) следующую задачу Коши, подобную (3.11):

$$
\partial_{t} X_{A}=-(i \hbar)^{-1}\left[F, X_{A}\right],\left.\quad X_{A}\right|_{t=0}=0,
$$

где

$$
X_{A}=\mathcal{H} \overleftarrow{\partial}_{A}-(i \hbar)^{-1}\left[\mathcal{H}, t \mathcal{V}_{A}\right]
$$


В силу стандартных аргументов из (3.25) следует, что

$$
X_{A}=0 \quad \text { для любого } t,
$$

так что при $t=1$ мы получаем уравнение (3.18). Аналогичные рассуждения применимы к любой наблюдаемой, подобной $\mathcal{H}$.

Отметим, что процедура рескейлинга, примененная к уравнениям (3.2) и (3.18), может быть обобщена следующим естественным образом. Вместо умножения соответствующего уравнения на $Z^{A}$ и затем рескейлинга (3.5) рассмотрим линию

$$
\partial_{s} \bar{Z}^{A}=\chi^{A}(\bar{Z}), \quad \bar{Z}^{A}(s=0)=Z^{A}, \quad \partial_{s}=\frac{\partial}{\partial s},
$$

где $\chi^{A}(\bar{Z})$ - некоторые регулярные функции. Например, если выбрать $\chi^{A}=\bar{Z}^{A}$, уравнение (3.27) дает

$$
\bar{Z}^{A}=Z^{A} e^{s},
$$

что в точности есть рескейлинг (3.5) с $t=e^{s}$. Теперь можно использовать общую линию (3.27) для определения обобщенной процедуры "рескейлинга" соответствующих уравнений. Именно, следует взять уравнение в точке $\bar{Z}$ и затем умножить его на $\partial_{s} \bar{Z}$. Например, применительно к уравнению (3.2) обобщенная процедура рескейлинга в точке $\bar{Z}$ дает

$$
\left(\partial_{s} \mathcal{V}_{A} G_{B}^{A}\right)-F \overleftarrow{\partial}_{A} G_{B}^{A}+(i \hbar)^{-1}\left[F, \mathcal{V}_{A} G_{B}^{A}\right]=0
$$

где $G_{B}^{A}$ и $F$ определены как

$$
\partial_{s} G_{B}^{A}=\chi^{A} \overleftarrow{\partial}_{C} G_{B}^{C}, \quad G_{B}^{A}(s=0)=\delta_{B}^{A}, \quad F=\mathcal{V}_{A} \chi^{A}
$$

Можно показать, что обобщенное линейное уравнение (3.29) предполагает соответствующий аналог задачи Коши (3.11), так что, если (3.29) удовлетворено, его решения удовлетворяют также (3.2).

В заключение заметим, что представление решения для $\mathcal{V}_{A}$ в форме Картана имеет следующую естественную реализацию:

$$
\mathcal{V}_{A}=(-i \hbar)\left(\partial_{A} U\right) U^{-1}(-1)^{\varepsilon_{A}} .
$$

Легко проверить, что решение (3.31) удовлетворяет (3.2). Если разрешить (3.31) для $U$, то получим $T$-упорядоченную экспоненту:

$$
U=T \exp \left\{\frac{i}{\hbar} \int \mathcal{V}_{A} d Z^{A}\right\} .
$$

Тогда из (3.31) выводим следующее уравнение ( $T^{*}$ означает антиупорядочивание):

$$
\mathcal{V}_{A}=T \exp \left\{\frac{i}{\hbar} \int \mathcal{V} d Z\right\} \overleftarrow{\partial}_{A}(-i \hbar) T^{*} \exp \left\{-\frac{i}{\hbar} \int \mathcal{V} d Z\right\}
$$

которое фактически является аналогом, в терминах контурного интеграла, уравнения нулевой кривизны (3.2). 
Уравнение (3.2), записанное для символов, имеет вид

$$
\left(\partial_{A} \mathcal{V}_{A}+\partial_{B} \mathcal{V}_{B}(-1)^{\left(\varepsilon_{A}+1\right)\left(\varepsilon_{B}+1\right)}\right)(-1)^{\varepsilon_{A}}+(i \hbar)^{-1}\left(\mathcal{V}_{A} \star \mathcal{V}_{B}-\mathcal{V}_{B} \star \mathcal{V}_{A}(-1)^{\varepsilon_{A} \varepsilon_{B}}\right)=0
$$

где «-умножение определяется в (3.14). Решение последнего уравнения можно искать в виде квазиклассического разложения по степеням $\hbar$. Низший порядок квантовой поправки к классическому уравнению (2.20) имеет вид

$$
\frac{1}{3}(i \hbar)^{2} \mathcal{V}_{A} \Delta^{3} \mathcal{V}_{B}
$$

где введено обозначение

$$
2 \Delta=\frac{\overleftarrow{\partial}}{\partial \Phi^{A}} \eta^{A B} \frac{\vec{\partial}}{\partial \Phi^{B}}
$$

Уравнение (3.18) переписывается для символов в виде

$$
\mathcal{H} \overleftarrow{\partial}_{A}-(i \hbar)^{-1}\left(\mathcal{H} \star \mathcal{V}_{A}-\mathcal{V}_{A} \star \mathcal{H}\right)=0
$$

При $\hbar=0$ из (3.37) имеем

$$
\mathcal{H} \overleftarrow{\partial}_{A}-\left\{\mathcal{H}, \mathcal{V}_{A}\right\}=0
$$

Квантовая поправка низшего порядка к классическому уравнению (3.38) есть

$$
-\frac{1}{3}(i \hbar)^{2} \mathcal{H} \Delta^{3} \mathcal{V}_{A}
$$

Формулы (3.35), (3.39) являются частными случаями специфического вида коммутатора символов Вейля,

$$
(i \hbar)^{-1}[F, G]_{\star}=(i \hbar)^{-1}\left(F \star G-G \star F(-1)^{\varepsilon_{F} \varepsilon_{G}}\right)=2(\hbar)^{-1} F \sin (\hbar \Delta) G,
$$

который следует из свойства антисимметрии оператора $\Delta(3.36)$.

В качестве явного примера того, как работают уравнения для символов, подобные (3.34) и (3.37), для обеспечения механизма генерации структурных соотношений алгебры конвертированных связей, мы представляем более детально процедуру разложений по $\hbar$ и $\Phi$ в случае уравнения (3.34). Исходим из следующего разложения по $\hbar$ и $\Phi$ для $\mathcal{V}_{A}$ :

$$
\mathcal{V}_{A}=\sum_{q=0}^{\infty}(i \hbar)^{q} \sum_{p=0}^{\infty} V_{A B_{p} \ldots B_{1}}^{(q)} \Phi^{B_{1}} \ldots \Phi^{B_{p}} .
$$

Соответствующая форма структурных соотношений для $V_{A B_{p} \ldots B_{1}}$ имеет вид

$$
\begin{aligned}
& \left(\partial_{A} V_{B B_{p} \ldots B_{1}}^{(q)}+\partial_{B} V_{A B_{p} \ldots B_{1}}^{(q)}(-1)^{\left(\varepsilon_{A}+1\right)\left(\varepsilon_{B}+1\right)}\right)(-1)^{\varepsilon_{A}}+ \\
& \quad+\sum_{k, l, m, n, s \geqslant 0} \delta_{k+l+s-1}^{q} \delta_{m+n}^{p} \frac{(m+s) !(n+s) !}{m ! s ! n !}(2)^{-s} \times \\
& \quad \times \operatorname{Sym}_{\left(B_{p} \ldots B_{1}\right)}\left[\left(V_{A B_{p} \ldots B_{n+1} C_{s} \ldots C_{1}}^{(k)} V_{B}^{(l) C_{1} \ldots C_{s}} B_{n} \ldots B_{1}(-1)^{\varepsilon_{B} \sum_{j=n+1}^{p} \varepsilon_{B_{j}}}\right)-\right. \\
& \left.\quad-(A \leftrightarrow B)(-1)^{\varepsilon_{A} \varepsilon_{B}}\right]=0,
\end{aligned}
$$


где введено обозначение

$$
\begin{aligned}
& V_{B}^{(l) C_{1} \ldots C_{s}}{ }_{B_{n} \ldots B_{1}}= \\
& \quad=\eta^{C_{1} D_{1}} \ldots \eta^{C_{s} D_{s}} V_{B D_{s} \ldots D_{1} B_{n} \ldots B_{1}}^{(l)}(-1)^{\sum_{i=1}^{s} \varepsilon_{D_{i}}\left(\sum_{j=i+1}^{s} \varepsilon_{C_{j}}+\varepsilon_{B}+1\right)} .
\end{aligned}
$$

Операция симметризации определяется как

$$
\begin{gathered}
\operatorname{Sym}_{\left(B_{p} \ldots B_{1}\right)}\left[X_{B_{p} \ldots B_{1}}\right]=X_{A_{p} \ldots A_{1}} S_{B_{p} \ldots B_{1}}^{A_{1} \ldots A_{p}} \\
p ! S_{B_{p} \ldots B_{1}}^{A_{1} \ldots A_{p}}=\Phi^{A_{1}} \ldots \Phi^{A_{p}} \overleftarrow{\partial}_{B_{p}} \ldots \overleftarrow{\partial}_{B_{1}}, \quad \partial_{A}=\frac{\partial}{\partial \Phi^{A}}
\end{gathered}
$$

В случае уравнения (3.37) процедура разложений по $\hbar$ и $\Phi$ является совершенно аналогичной (с точностью до элементарных модификаций) тому, что было сделано в случае уравнения (3.34). Будем исходить из следующего разложения по $\hbar$ и $\Phi$ для $\mathcal{H}$ :

$$
\mathcal{H}=\sum_{q=0}^{\infty}(i \hbar)^{q} \sum_{p=0}^{\infty} H_{B_{p} \ldots B_{1}}^{(q)} \Phi^{B_{1}} \ldots \Phi^{B_{p}} .
$$

Тогда полный набор структурных соотношений для $H_{B_{p} \ldots B_{1}}^{(q)}$ представляется в виде

$$
\begin{aligned}
& \partial_{A} H_{B_{p} \ldots B_{1}}^{(q)}(-1)^{\varepsilon_{A}}+\sum_{k, l, m, n, s \geqslant 0} \delta_{k+l+s-1}^{q} \delta_{m+n}^{p} \frac{(m+s) !(n+s) !}{m ! s ! n !}(2)^{-s} \times \\
& \times \operatorname{Sym}_{\left(B_{p} \ldots B_{1}\right)}\left[V_{A B_{p} \ldots B_{n+1} C_{s} \ldots C_{1}}^{(k)} H_{B_{n} \ldots B_{1}}^{(l) C_{1} \ldots C_{s}}-\right. \\
& -H_{B_{p} \ldots B_{n+1} C_{s} \ldots C_{1}}^{(k)} V_{A}^{(l) C_{1} \ldots C_{s} \ldots B_{1}}(-1)^{\left.\varepsilon_{A} \sum_{i=n+1}^{p} \varepsilon_{B_{i}}\right]}=0,
\end{aligned}
$$

где мы обозначили аналог (3.43)

$$
H_{B_{n} \ldots B_{1}}^{(l) C_{1} \ldots C_{s}}=\eta^{C_{1} D_{1}} \ldots \eta^{C_{s} D_{s}} H_{D_{s} \ldots D_{1} B_{n} \ldots B_{1}}^{(l)}(-1)^{\sum_{i=1}^{s} \varepsilon_{D_{i}}\left(\sum_{j=i+1}^{s} \varepsilon_{C_{j}}+1\right)} .
$$

Наконец, напомним определение внутреннего звездочного криволинейного произведения в терминах символов Вейля [17]. Пусть $\mathcal{F}, \mathcal{G}$ суть две наблюдаемые

$$
\left[T_{A}, \mathcal{F}\right]_{\star}=0,\left.\quad \mathcal{F}\right|_{\Phi=0}=F, \quad\left[T_{A}, \mathcal{G}\right]_{\star}=0,\left.\quad \mathcal{G}\right|_{\Phi=0}=G
$$

где $T_{A}=P_{A}-\mathcal{V}_{A}$ являются абелевыми связями после конверсии. Тогда внутреннее криволинейное звездочное произведение $\star_{\text {D }}$ определяется как

$$
F \star_{D} G=\left.(\mathcal{F} \star \mathcal{G})\right|_{\Phi=0} .
$$

Это звездочное произведение ассоциативно, но не коммутативно. Соответствующий звездочный коммутатор имеет вид

$$
[F, G]_{\star_{\mathrm{D}}}=F \star_{\mathrm{D}} G-G \star_{\mathrm{D}} F(-1)^{\varepsilon_{F} \varepsilon_{G}} .
$$

В классическом пределе коммутатор (3.50) точно воспроизводит нелинейную скобку Пуассона (2.15),

$$
\left.(i \hbar)^{-1}[F, G]_{\star_{\mathrm{D}}}\right|_{\hbar \rightarrow 0}=\{F, G\}_{\mathrm{D}}
$$


Конечно, каждый из символов $\mathcal{F}, \mathcal{G}$ может быть разложен в степенной ряд, подобный (3.45), чтобы удовлетворить соответствующему набору структурных соотношений (3.46). Затем можно вывести соответствующее разложение по $ћ$ для внутреннего криволинейного звездочного произведения (3.49):

$$
\begin{aligned}
& F \star_{\mathrm{D}} G=\sum_{q \geqslant 0}(i \hbar)^{q} \sum_{k, l, s \geqslant 0} \delta_{k+l+s}^{q} s !(2)^{-s} \times \\
& \times F_{C_{s} \ldots C_{1}}^{(k)} \eta^{C_{1} D_{1}} \ldots \eta^{C_{s} D_{s}} G_{D_{s} \ldots D_{1}}^{(l)}(-1)^{\sum_{i=1}^{s} \varepsilon_{D_{i}}\left(\sum_{j=i+1}^{s} \varepsilon_{C_{j}}+\varepsilon_{G}+1\right)} .
\end{aligned}
$$

\section{4. ВМЕСТО ЗАКЛЮЧЕНИЯ}

Отметим, что в настоящей статье мы ограничились рассмотрением только тех аспектов, которые тесно связаны с формальным решением условия нулевой кривизны для расширенного симплектического потенциала и соответствующей проблемы для гамильтониана и других физических наблюдаемых. Здесь мы не рассматривали аспекты, связанные с гильбертовым пространством БФФ (Баталина-ФрадкинаФрадкина) конвертированной системы [13]-[15]: представление Фока, упорядочивание Вика и физические состояния. Мы также не рассматривали топологические аспекты глобального описания, тесно связанные со структурой расслоения симплектических спиноров и метаплектической аномалии. Детальное рассмотрение этих аспектов можно найти в превосходном обзоре Фрадкина и Линецкого [17]. Дополнительную информацию по топологическим аспектам геометрического квантования можно найти в прекрасной книге Карасева и Маслова [23].

Благодарности. И.А. Баталин благодарен Клаусу Берингу из университета Масарика за интересные обсуждения.

\section{Список литературы}

[1] F. A. Berezin, Commun. Math. Phys., 40:2 (1975), 153-174.

[2] D. J. Simms, N. M. J. Woodhouse, Lectures on Geometric Quantization, Lecture Notes in Physics, 53, Springer, Berlin, 1976.

[3] N. M. J. Woodhouse, Rep. Math. Phys., 12:1 (1977), 45-56.

[4] M. Forger, H. Hess, Commun. Math. Phys., 64:3 (1979), 269-278.

[5] N. M. J. Woodhouse, Geometric Quantization, Oxford Univ. Press, New York, 1992.

[6] E. S. Fradkin, G. A. Vilkovisky, Phys. Lett. B, 55:2 (1975), 224-226.

[7] I. A. Batalin, G. A. Vilkovisky, Phys. Lett. B, 69:3 (1977), 309-312.

[8] B.V. Fedosov, Deformation Quantization and Index Theory, Mathematical Topics, 9, Akademie, Berlin, 1996.

[9] M. Alexandrov, M. Kontsevich, A. Schwarz, O. Zaboronsky, Internat. J. Modern Phys. A, 12:7 (1997), 1405-1429, arXiv: hep-th/9502010.

[10] A. S. Cattaneo, G. Felder, Commun. Math. Phys., 212:3 (2000), 591-611, arXiv: math/ 9902090.

[11] I. A. Batalin, E. S. Fradkin, Phys. Lett. B, 180:1-2 (1986), 157-164.

[12] I. A. Batalin, E. S. Fradkin, Nucl. Phys. B, 279:3 (1987), 514-528.

[13] I. A. Batalin, E. S. Fradkin, T. E. Fradkina, Nucl. Phys. B, 314:1 (1989), 158-174.

[14] I. A. Batalin, E. S. Fradkin, Nucl. Phys. B, 326:3 (1989), 701-718.

[15] I. A. Batalin, E. S. Fradkin, T. E. Fradkina, Nucl. Phys. B, 332:3 (1990), 723-736.

[16] E. S. Fradkin, V. Ya. Linetsky, Nucl. Phys. B, 444:3 (1995), 577-601. 
[17] E. S. Fradkin, V. Ya. Linetsky, Nucl. Phys. B, 431:3 (1994), 569-621.

[18] M. A. Grigoriev, S. L. Lyakhovich, Commun. Math. Phys., 218:2 (2001), 437-457, arXiv: hep-th/0003114.

[19] И. А. Баталин, М. А. Григорьев, С. Л. Ляхович, ТМФ, 128:3 (2001), 324-360.

[20] I. Batalin, M. Grigoriev, S. Lyakhovich, J. Math. Phys., 46:7 (2005), 072301, 16 pp.

[21] I. A. Batalin, E. S. Fradkin, Modern Phys. Lett. A, 4:11 (1989), 1001-1011.

[22] I. A. Batalin, I. V. Tyutin, Internat. J. Modern Phys. A, 6:18 (1991), 3255-3282.

[23] М. В. Карасев, В. П. Маслов, Нелинейнъе скобки Пуассона. Геометрия и квантование, Наука, М., 1991. 